UDC 004.052

\title{
TRANSIENT PROCESS AT LOW-ORDER FREQUENCY DEPENDENT DIGITAL COMPONENTS
}

Ukhina A. V. - Post-graduate student, Odessa National Polytechnic University Computing systems Department, Odessa, Ukraine.

Sitnikov V. S. - Dr. Sc., Professor, Odessa National Polytechnic University Computing systems Department, Odessa, Ukraine.

ABSTRACT

Context. When constructing specialized and programmable mobile systems, there arises the problem of system restructuring, under system functioning conditions breach because of changes in the noise-signaling environment or the system operating conditions. Any rearrangement of the system's digital frequency-dependent components leads to the occurrence of transient process, which duration is determined by the components characteristics. The traditional approach to the transient process analysis refers to the zero initial conditions, however, the intelligent sensors and specialized computer systems operation as well as parameters adjustment as well as other system components can be performed under non-zero initial conditions. This implies the need for a large number of computations, not feasible sometimes in real time. Here essential is to assess the duration of process' transition to readjustment process to provide the system operability under new conditions.

Objective. To estimate the transient process' maximum duration when rearrangement, to determine the possible rearrangements range and width, taking into account the system stability.

Method. This research carrying out improved is the indirect method of transient process duration estimating by the transfer function poles simplifying the irrational function expansion.

Results. The effected analysis with relevant modeling and theoretical verification allowed obtaining relations to estimate the transient process maximum duration and to determine possible modifications range and width taking into account the system stability. Upon research results we built the dependencies of transient process duration onto the cutoff respective frequency. Simplified is the representation of relation used to determine the transient process length at the expense of decomposing into order series.

Conclusions. The results obtained allow us to estimate the transient process duration upper limit by improving the indirect method of estimating the transient process duration along the transfer function poles, while simplifying the irrational function expansion, that making possible, before the rearrangement beginning, considering the given new relative cutoff frequency and the component order, to predict the component stability afterwards. From a practical point of view, this reduces the calculations amount and due to the predicted result, increases the specialized computer system overall and by-components efficiency for specified performance criteria. The results obtained are applicable to the design of computer systems' microprocessor components.

KEYWORDS: filtering, intelligent sensors, transient process duration analysis, filter parameters rearrangement.

\author{
ABBREVIATIONS \\ DTC - discrete transient characteristics; \\ LO FDDC - low-order frequency-dependent digital \\ components; \\ LPF - low-pass filter.
}

\section{NOMENCLATURE}

$t_{\Pi \Pi}-$ transient process time;

$a . b$ - real coefficients;

$N$ - transient process duration;

A - constant depending on the initial conditions at an equivalent relative damping constant $\tau$;

$\beta$ - complex root modulus;

$\tau$ - relative damping constant;

$\bar{\omega}_{c}-$ relative cutoff frequency;

$\mathrm{H}(\mathrm{z})$ - transfer function;

$\mathrm{T}$ - quantification period;

$f_{d}$ - linear discretisation frequency;

$f$ - linear cutoff frequency;

$\mathrm{z}$ - complex variable;

$\Delta-$ some given number.

\section{INTRODUCTION}

A rapid microprocessor equipment development, its computational power growth, along to the cost decrease,

(C) Ukhina A. V., Sitnikov V. S., 2018

DOI $10.15588 / 1607-3274-2018-3-5$ enabled modern microprocessors' incorporation into various specialized computer systems components. The combination of a measuring transducer and a microprocessor at such systems allows performing basic operations to transform and increase the measurement information reliability at those data origination point [1-6]. Such sensors are called smart sensors.

In accordance with the Fieldbus Foundation ideology and $t$ standard, typical algorithms for measurement information processing (filtration, scaling, linearization, etc.), control and regulation are transferred to the lowest control level; this one of intelligent sensors and actuators [1, 4-6]. For example, the choice of filters and their parameters in accordance with the interference characteristics is based on the sensor signal analysis or the control command.

Such situation involves the need to develop adjustable LO FDDC, because their connection considering it is possible to construct a FDDC of any order. It should be noted that the FDDC concept is broader than this one of digital filter. Such structure of an adjustable high-order digital filter allows much easier tuning and rearrangement $[7,8]$.

\section{PROBLEM STATEMENT}

The operating conditions at an intelligent sensor may vary. When some operating conditions change the filter shall readjust to control these changes in accordance with the specified criterion. If the changes are slow, i.e. occur 
over time exceeding the filter transient process duration, the filter, as a rule, is tracking them $[9,10]$. The change speed increasing the adjustable filter efficiency will decrease, because the filter does not have time enough to fully retune during that change time (in this case, its transient process has not been ended).

Then, there arises the problem of analyzing the transient process duration and type, as well as estimating the similar LO FDDCs; transient process maximum duration when the transfer functions coefficients are rearranged.

\section{REVIEW OF THE LITERATURE}

It is known from the systems theory that the transient process duration is most often determined by the transfer function root at minimal real part [11-15]. A classical approach to the transient process duration analysis of the implies zero initial conditions. However, the operation of intelligent sensors, specialized computer systems, and parameters adjustment can occur even under non-zero initial conditions. Such an analysis is rather complicated and requires large calculations with different variants of the transient process, which sometimes is not feasible in real time.

When creating intelligent sensors and specialized computer systems, which include a large number of components, such an analysis is rather problematic. Therefore, several authors suggest setting, at the design stage, the system critical frequency, that shall be higher than the frequency of possible interference or effects on the system $[11,16]$.

In addition, it is proposed to estimate not the time of the transient itself, but only the exponential time constant, serving to approximate the transient process or time of the studied transient process [16].

When engineering analysis of the system, it is proposed to take as the first approximation departing point the transient process duration at zero initial conditions [17].

Since basically systems operate under non-stationary conditions, several foreign authors suggest analyzing the quasi-stationary duration, for example, based on statistical linearization methods. In this case, the quasi-stationary regions should overpass the system transient process duration [18].

All these approaches determine the range of possible changes or rearrangements when system adaptation or restructuring takes place.

Works $[19,20]$ expose results of transient processes' analysis for digital automatic control systems.
Usually, the transient process quality is estimated by direct or indirect indicators [12]. Direct indicators characterize immediately the transition process itself, as a response to a typical impact (usually stepwise). Indirect indicators evaluate the transient process by locating the characteristic equation roots or the poles of the transfer function, according to the system frequency characteristics or integral estimates. In general, the transient process quality is affected by both the transfer function's poles and zeros location.

\section{MATERIALS AND METHODS}

To analyse the transient process, we use the discrete transition characteristic (DTC) influenced by the $\delta$-pulse, thus analysing the transient process free component and the single step response, as well as the studied transient process forced component [10, 20-21].

The transient process duration is defined as the time interval from the time, e.g., the stepwise action has been applied to the time point after which the deviations of the output value from its new steady-state cipher become less than a certain predetermined number $\Delta$. In general, this value is determined by the device task and condition, but usually this cipher is taken at a level $5 \%$ of the steadystate value. Depending on the device, such time is specified as the number of output value oscillations or as (4-5) $\tau$, where $\tau$ is the relative attenuation constant. Therefore, everything depends on the type of transient process: oscillatory, aperiodic or monotonic [12, 19, 20].

\section{RESULTS}

Let us consider the effect on the first-order and second-order FDDC transient process duration and type on the example of the low and high pass filters transfer functions when the relative cut-off frequency $\bar{\omega}_{c}$ changes on the segment, $\bar{\omega}_{c}=[0.1 \div 0.9]$, where $\bar{\omega}_{c}=\frac{2 \pi \frac{f}{f_{d}}}{\pi}$, $\bar{\omega}_{c} \in[0 \div 1]$.

The transient process duration was determined in relative units: cycles, as $N=t_{\Pi \Pi} \cdot f_{d}$. Such representation of the result allows comparing different FDDCs at different sampling rates.

The analysis results are given in Tables $1-4$, showing the relative frequency, transfer function poles, and the components' transient process duration in cycles.

Table 1 - Duration of the first-order FDDC transient process free component

\begin{tabular}{|l|l|l|l|l|l|l|}
\hline $\bar{\omega}_{c}$ & 0.1 & 0.26 & 0.42 & 0.58 & 0.74 & 0.9 \\
\hline$\beta$ & 0.7265 & 0.3959 & 0.1263 & -0.1263 & -0.3959 & -0.7265 \\
\hline HP & 7 & 5 & 4 & 4 & 5 & 7 \\
\hline LP & 7 & 5 & 4 & 4 & 5 & 7 \\
\hline
\end{tabular}

Table 2 - Duration of the first-order FDDC transient process forced component

\begin{tabular}{|c|c|c|c|c|c|c|}
\hline $\bar{\omega}_{c}$ & 0.1 & 0.26 & 0.42 & 0.58 & 0.74 & 0.9 \\
\hline$\beta$ & 0.7265 & 0.3959 & 0.1263 & -0.1263 & -0.3959 & -0.7265 \\
\hline $\mathrm{HP}$ & 11 & 5 & 4 & 4 & 4 & 6 \\
\hline $\mathrm{LP}$ & 11 & 5 & 4 & 4 & 4 & 6 \\
\hline
\end{tabular}


Table 3 - Duration of the second-order FDDC transient process free component

\begin{tabular}{|c|c|c|c|c|c|c|}
\hline $\bar{\omega}_{c}$ & 0.1 & 0.26 & 0.42 & 0.58 & 0.74 & 0.9 \\
\hline $\mathrm{Z}$ & $0.7805 \pm 0.1793 \mathrm{i}$ & $0.4517 \pm 0.3401 \mathrm{i}$ & $0.1476 \pm 0.4065 \mathrm{i}$ & $-0.1476 \pm 0.4065 \mathrm{i}$ & $-0.4517 \pm 0.3401 \mathrm{i}$ & $-0.7805 \pm 0.1793 \mathrm{i}$ \\
\hline $\mathrm{HP}$ & 11 & 6 & 6 & 6 & 4 & 7 \\
\hline $\mathrm{LP}$ & 7 & 7 & 5 & 6 & 6 & 11 \\
\hline
\end{tabular}

Table 4 - Duration of the second-order FDDC transient process forced component

\begin{tabular}{|c|c|c|c|c|c|c|}
\hline $\bar{\omega}_{c}$ & 0.1 & 0.26 & 0.42 & 0.58 & 0.74 & 0.9 \\
\hline $\mathrm{z}$ & $0.7805 \pm 0.1793 \mathrm{i}$ & $0.4517 \pm 0.3401 \mathrm{i}$ & $0.1476 \pm 0.4065 \mathrm{i}$ & $-0.1476 \pm 0.4065 \mathrm{i}$ & $-0.4517 \pm 0.3401 \mathrm{i}$ & $-0.7805 \pm 0.1793 \mathrm{i}$ \\
\hline $\mathrm{HP}$ & 11 & 8 & 6 & 5 & 4 & 6 \\
\hline $\mathrm{LP}$ & 16 & 7 & 5 & 4 & 6 & 9 \\
\hline
\end{tabular}

\section{EXPERIMENTS}

To analyze and evaluate the transient process duration, a simulative modeling was carried out with the mathematical package SciLab.

In Fig. 1 and Fig. 2 shown is the free component transient process type when the $\delta$-pulse is applied to the first and second order low-pass filters.

It can be seen from the figures that, for relative cut-off frequency small values, the transient process is aperiodic, and for relative cut-off frequency large values the process is oscillatory with a damped amplitude.

Based on the above, the dependence between transient process duration and the relative cutoff frequency is plotted, Fig. 3 and Fig. 4.

The obtained data allow estimating the transient process maximum duration to select the range of possible effects on the component operation and the component transfer function coefficients adjustment range.

\section{DISCUSSION}

Theoretical research and simulative modeling showed that to estimate the transient process duration upper limit, the relations proposed in [11-12] can be modified.

For example, in [12] the author shows that the transient process duration is determined only by the complex root module $\beta$ or by the module of the bigger (from among two) real root. The process discrete values at the points of time quantization will be located inside the region bounded by the symmetric envelopes

$$
\varphi(t)= \pm A e^{-\frac{\ln (\beta t)}{T}},
$$

where $T=\frac{1}{f_{d}}-$ quantization period, A - constant, depending on the initial conditions, containing an equivalent relative damping constant $\tau$. In accordance with these provisions, obtained is the theoretical curve shown in Fig. 3 and Fig. 4.
The results obtained in a generalized form for digital systems can be represented by the relation [21]

$$
N=\frac{a}{|\ln (\beta)|}+b,
$$

where $a . b$ - real coefficients $(1<a<3,2<b<4), \beta-$ complex root module or module of the bigger real root, $0<\beta<1$.

However, in this form the obtained ratio is difficult to use at microprocessor and computer systems. Therefore, we replace $\ln (\beta)$ by expanding into power series [23, 24]. Then transforming and simplifying, we obtain the following representation

$$
-\ln (\beta)=2 \frac{1-\beta}{1+\beta}\left[1+\frac{(1-\beta)^{2}}{3(1+\beta)^{2}}+\frac{(1-\beta)^{4}}{5(1+\beta)^{4}}\right] .
$$

After modeling and experimental verification, it is possible to relay onto the first term

$$
-\ln (\beta)=2 \frac{1-\beta}{1+\beta}
$$

In this case, relation (1), taking into account the simplification, can be represented as follows

$$
N=\frac{a}{2} \cdot \frac{1+\beta}{1-\beta}+b .
$$

Graphically the theoretical dependence between of the transient process duration upper limit in cycles that shall be $N=f\left(\bar{\omega}_{c}\right)$, where $\bar{\omega}_{c}$ - the relative cutoff frequency, determined by the component transfer function coefficients $a$ and $b$ has the form shown in Fig. 5. 


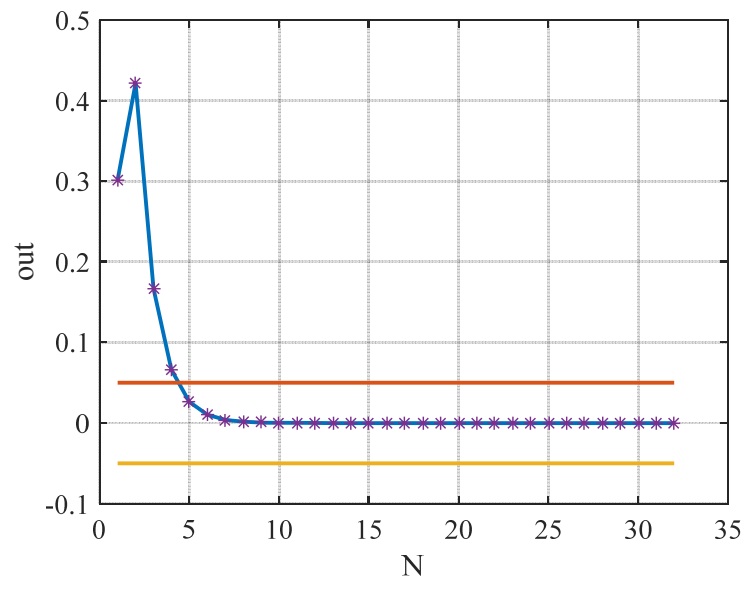

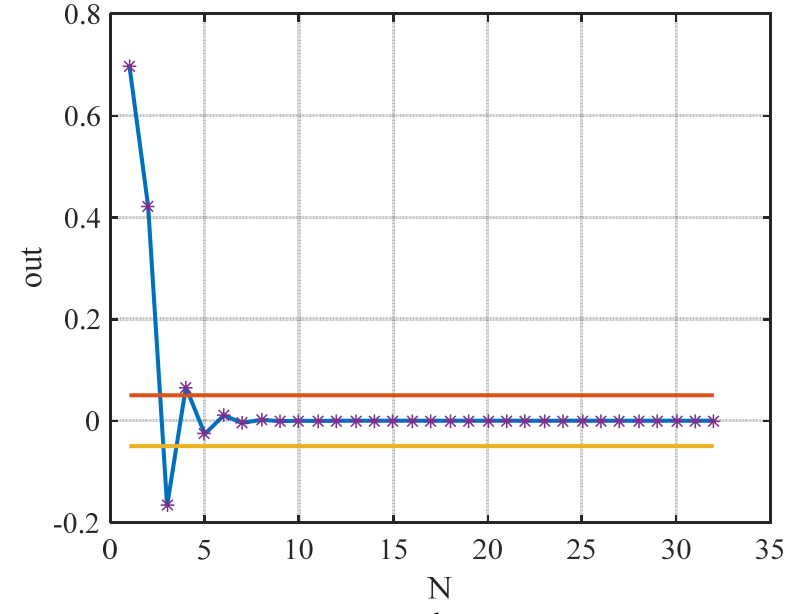

$\mathrm{b}$

Figure $1-1^{\text {st }}$ order low-pass filter transient process at: $\mathrm{a}-\bar{\omega}_{C}=0.26, \beta=0.3959 ; \mathrm{b}-\bar{\omega}_{C}=0.74, \beta=-0.3959$

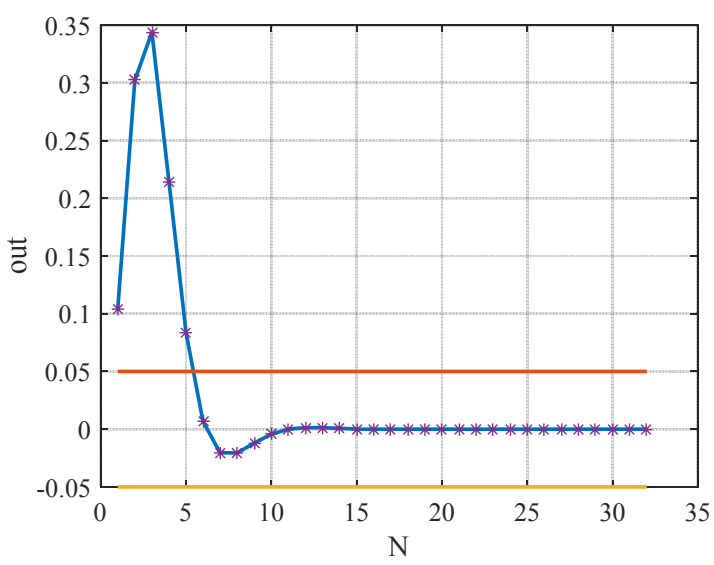

a

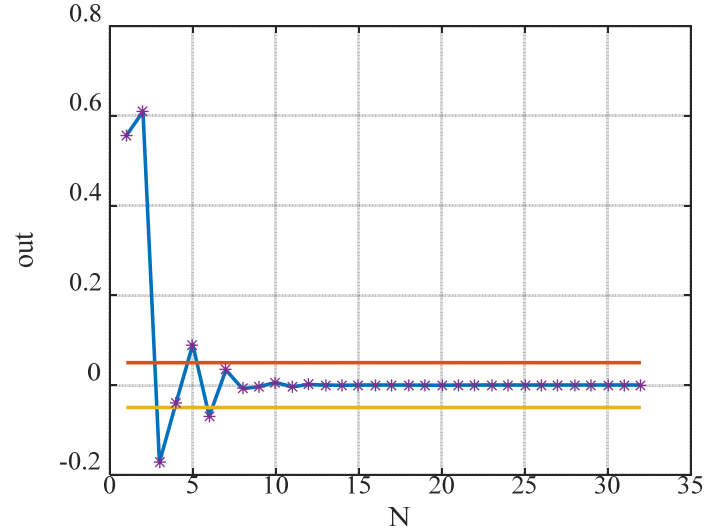

b

Figure $2-2^{\text {nd }}$ order low-pass filter transient process at: $\mathrm{a}-\bar{\omega}_{c}=0.26, \mathrm{z}=0.4517 \pm 0.3401 i ; \mathrm{b}-\bar{\omega}_{c}=0.74, \mathrm{z}=-0.4517 \pm 0.3401 i$

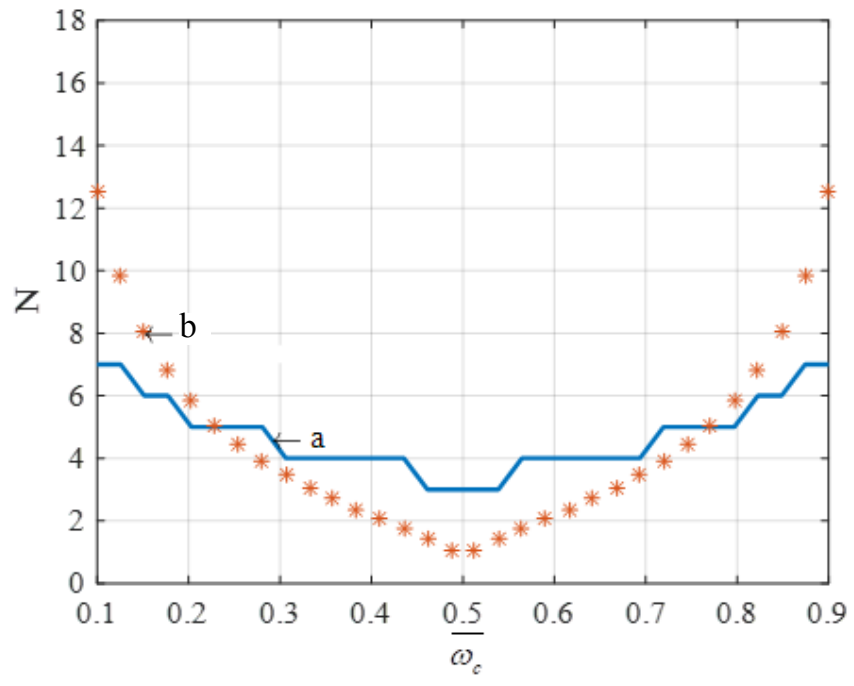

Figure $3-1^{\text {st }}$ order low-pass filter transient process duration dependency on the relative cut-off frequency $\bar{\omega}_{\mathcal{C}}$ : $\mathrm{a}$ - experimental; $\mathrm{b}$ - theoretical 


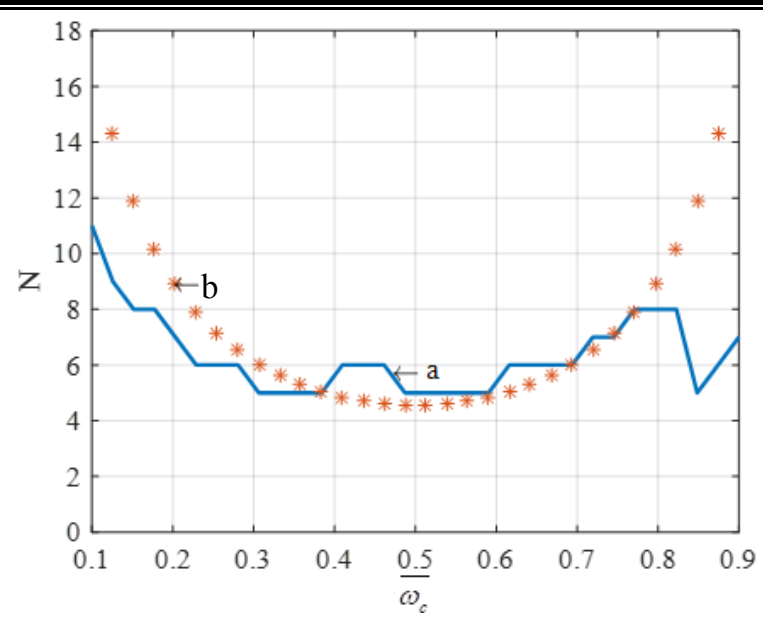

Figure $4-2^{\text {nd }}$ order low-pass filter transient process duration dependency on the relative cut-off frequency $\bar{\omega}_{c}$ :

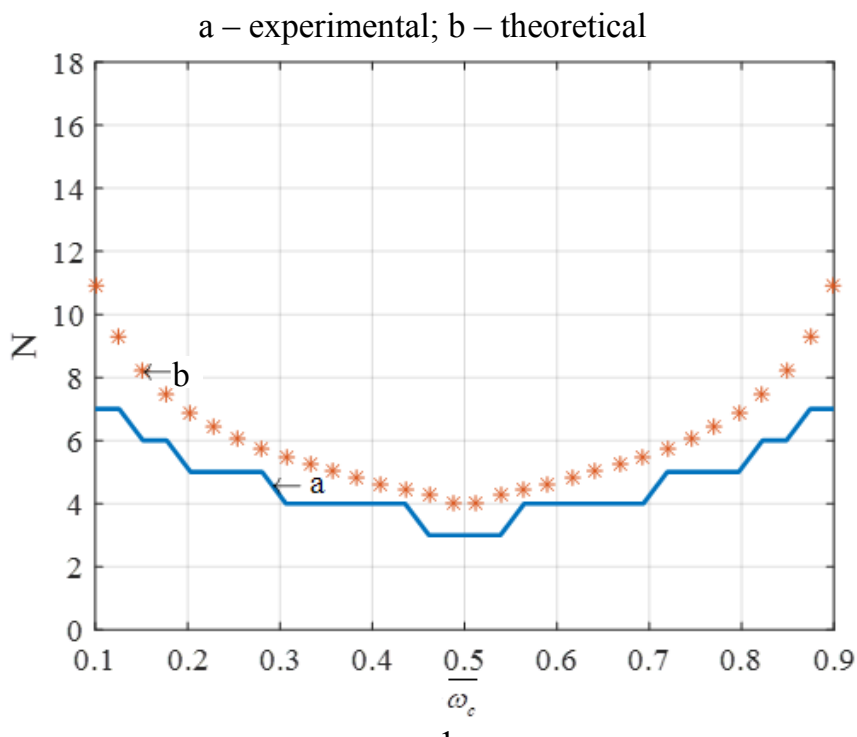

1

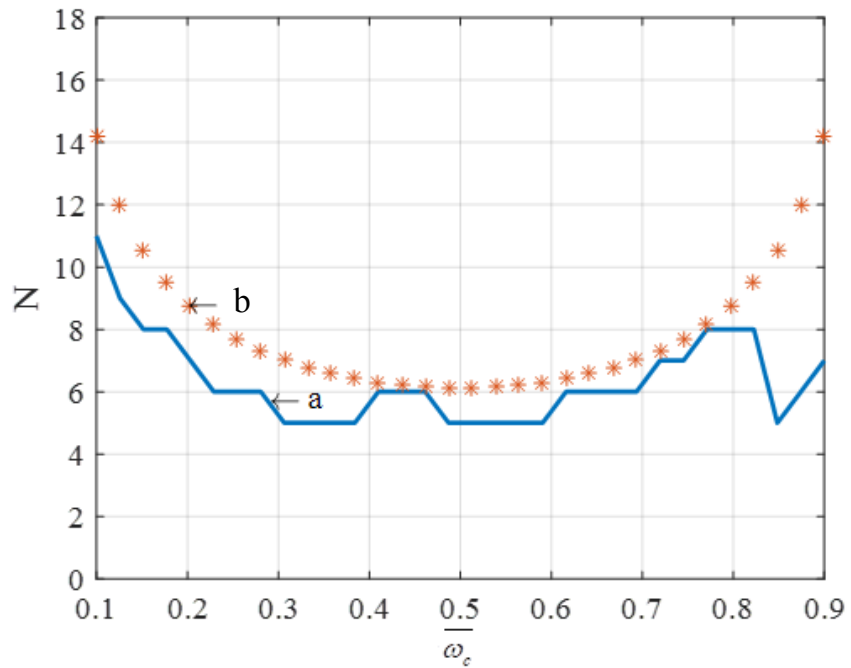

2

Figure 5 - Graph of the transient process duration upper limit for the frequency-dependent component

$$
1-1^{\text {st }} \text { order type } H(z)=\frac{a_{0}+a_{1} z^{-1}}{1+b_{1} z^{-1}} ; 2-2^{\text {nd }} \text { order type } H(z)=\frac{a_{0}+a_{1} z^{-1}+a_{2} z^{-2}}{1+b_{1} z^{-1}+b_{2} z^{-2}} \text {; }
$$

$\mathrm{a}$ - experimental; $\mathrm{b}$ - theoretical 


\section{CONCLUSIONS}

At the issue of analysis, simulation and experimental verification carried out, we obtained relationships allowing us to estimate the transition process upper limit as a function of the relative cut-off frequency and the frequency-dependent component. Such a representation of the transient process duration upper limit estimation renders possible to analyze the of the specialized computer system components capacities when their parameters or transfer function coefficients and whole system properties undergo changes, therefore to determine the possible rearrangements ranges and widths, taking into account the system stability. This is especially important when adjusting the coefficients of frequency-dependent components in adaptive systems. Results obtained therefore are applicable to the design of specialized computer systems adjustable frequency-dependent components, for example, an ultrasonic rangefinder [6].

\section{REFERENCES}

1. Borisova L. F. Metody analiza i rascheta perehodnykh protsessov v elektricheskikh tsepyakh, Mezhdunarodnyi zhurnal prikladnykh i fhundamentalnykh issledovanii, 2014, No. 6 , pp. 113-116.

2. Borodianskii I. M., Turulin I. I. Primenenie rekursivnykh KIKh-filtrov dlya podavleniya pomekh pri avtomaticheskom kontrole soprotivleniya izolyatsii, Izvestiya YuFU. Tekhnicheskie nauki, 2016, No. 10(183), pp. 99-110.

3. Voitovich I.D, Korsunskii, V.M. Intellektualnye sensory. Moscow, Internet-Universitet Informatsionnykh Tekhnologii; BINOM. Laboratoriia znanii, 2011, 624 p.

4. Tuzlukov V. Signal Processing in Radar Systems. CRC Press, 2013, 596 p

5. Kocon S., Piskorowski J. Digital finite impulse response notch filter with non-zero initial conditions, based on an infinite impulse response prototype filter, ASC Publications Anal. Chem, 2014, 86, pp. 3508-3516. dx.doi.org/10.1021/ac404150d.

6. Ukhina A.V, Bilenko A.A, Sitnikov V.S. Povyshenie effektivnosti programmno-tekhnicheskikh komplekcov v ASU TP AES. Yadernaya i radiotsionnaya bezopasnost, 2016 , No. 3, pp. 70-76.

7. Popov D. I. Analiz tsifrovykh sisitem obrabotki signalov. Izvestiya vysshikh uchebnykh zavedenii. Tekhnicheskie nauki, 2016, No. 2(18), pp. 83-92

8. Sytnikov V. S, Stupen P. V., Piven B. O. Vplyv osoblyvostei kaskadiv tsyfrovogo filtru vysokogo poryadku na vykhidnyi shum kvantovannya, Elektrotekhnicheskie $i$ kompiuternye sistemy, 2013, No. 9(85), pp. 97-101.
9. Dzhigan, V. Adaptivnye filtry i ikh prilozheniya $\mathrm{v}$ radiotekhnike i svyazi, Sovremennaya elektronika, 2013, No. 9, pp. 56-63.

10. Dorf R. C, Bishop R. H. Modern Control Systems. Prentice Hall, NJ 07458, 2001, 832 p.

11. Besekerskii V. A, Popov E. P. Teoriya sistem avtomaticheskogo regulirovaniya. Moscow, Nauka, 1975, $768 \mathrm{p}$.

12. Kuznetsov B.I, Sergeev V.E, Chernyshev V.M. Mikroprotsessornoe upravlenie mnogokanalnymi sistemami vysokoi tochnosti. Kiev, Technika, 1990, 208 p.

13. Ivanov B. A, Yushchenko A. S. Teoriya diskretnykh system avtomaticheskogo upravleniya. Moscow, Nauka, 1983, $336 \mathrm{p}$.

14. Kuo B. C. Digital Control Systems. Holt, Rinehart and Winston, Inc., 1980, 448 p.

15. Iserman R. Digital Control Systems. Springer-Verlag, Berlin, Heidelberg, N. York, 1981, 541p.

16. Anderson B.D.O, Bitmead R. R., Johnson C. R. Stability of Adaptive Systems: passivity and averaging analysis. The MIT Press Cambridge, Massachusetts, London, England, 1986, $263 \mathrm{p}$

17. Schulze K-P, Rehberg K-J. Entwurf von adaptiven Systemen Eine Darstellung fur Ingenieure. Veb Verlag Technik, Berlin, 1990, $448 \mathrm{p}$

18. Prikladnye matematicheskie metody analiza $\mathrm{v}$ radiotekhnike Ed. by G. V. Obrezkova. Moscow, Vyssh. Shk., 1985, $343 \mathrm{p}$.

19. Ed. by K. P. Pupkova, N. D. Egupova Metody klassicheskoi i sovremennoi teorii avtomaticheskogo upravleniya. Matematicheskie modeli, dinamicheskie kharakteristiki i analiz system avtomaticheskogo upravleniya. Moscow, Izd-vo MGTUim. N.E. Baumana, 2004, 654 p.

20. Zhuravlev A. Yu, Pavlov A. V, Zhurba V. O. Metodika povysheniya obshchei ustoichivosti i korrektsii pryamykh pokazatelei kachestva perekhodnykh protsessov $\mathrm{v}$ tsifrovykh sistemakh upravleniya, Kompressionnoe i energeticheskoe mashinostroenie, 2012, No. 1, pp. 36-40.

21. Kisel A. G, Sitnikov V. S, Ukhina A. V. Analiz dlitelnosti perekhodnogo protsessa pri nenulevykh nachalnykh usloviyakh. Problemy informatyky ta kompiuternoi tekhniky, 2017, pp. 100-102.

22. Ukhina A. V, Sitnikov V. S, Sitnikova V. A. Primenenie komponentov spetsializirovannykh sistem pri provedenii eksperimentalnykh medikobiologicheskikh issledovanii. «Datchyky, prylady ta systemy-2017», 2017, pp. 39-41.

Received 19.02.2018. Accepted 03.04.2018

\section{УДК 004.052}

\section{ПЕРЕХІДНИЙ ПРОЦЕС У ЦИФРОВИХ ЧАСТОТНО-ЗАЛЕЖНИХ КОМПОНЕНТАХ НИЗЬКОГО ПОРЯДКУ}

Ухіна Г. В. - аспірант кафедри «Комп’ютерні системи» Інституту комп’ютерних систем Одеського національного політехнічного університету, Одеса, Україна.

Ситніков В. С. - д-р техн. наук, професор кафедри «Комп’ютерні системи» Одеського національного політехнічного університету, Одеса, Україна.

\section{АНОТАЦІ}

Актуальність. При побудові спеціалізованих і програмованих мобільних систем виникає завдання перебудови системи, коли порушуються умови функціонування системи через зміни помехо-сигнального оточення або умов роботи системи. 
Будь-яка перебудова цифрових частотно-залежних компонент системи призводить до виникнення в системі перехідного процесу, тривалість якого визначається характеристиками компонент. Традиційний підхід до аналізу перехідного процесу здійснюється при нульових початкових умовах, однак робота інтелектуальних датчиків, спеціалізованих комп'ютерних систем і перебудова параметрів може здійснюватися при ненульових початкових умовах. Це призводить до необхідності великого числа обчислень, що іноді не можливо у реальному масштабі часу. У цих умовах необхідно оцінити тривалість перехідного процесу до процесу перебудови, щоб забезпечити роботу системи до нової перебудови.

Ціль. Оцінити максимальну тривалість перехідного процесу, визначити діапазон і ширину можливих перебудов з урахуванням забезпечення стійкості системи.

Метод. У роботі вдосконалено непрямий метод оцінки тривалості перехідного процесу по полюсах передавальної функції за рахунок спрощення розкладання іраціональної функції.

Результати. Проведений аналіз, моделювання і теоретична перевірка дозволили отримати співвідношення для оцінки максимальної тривалості перехідного процесу, визначити діапазон і ширину можливих перебудов 3 урахуванням забезпечення стійкості системи. Знайдена залежність виду перехідного процесу від відносної частоти зрізу. Побудовано залежності тривалості перехідного процесу від відносної частоти зрізу. Спрощено представлення співвідношення для визначення тривалості перехідного процесу за рахунок розкладання в ступеневий ряд.

Висновки. Отримані результати дозволяють оцінити верхню межу тривалості перехідного процесу, можливості компоненти спеціалізованої комп’ютерної системи при зміні параметрів, при цьому забезпечивши їі стійкість. Результати застосовують при проектуванні мікропроцесорних компонент комп'ютерних систем.

КЛЮЧОВІ СЛОВА: фільтрація, інтелектуальні датчики, аналіз тривалості перехідного процесу, перебудова параметрів фільтру.

\section{УДК 004.052}

\section{ПЕРЕХОДНОЙ ПРОЦЕСС В ЦИФРОВЫХ ЧАСТОТНО-ЗАВИСИМЫХ КОМПОНЕНТАХ НИЗКОГО ПОРЯДКА}

Ухина А. В. - аспирант кафедры «Компьютерные системы» Института компьютерных систем Одесского национального политехнического университета, Одесса, Украина.

Ситников В. С. - д-р техн. наук, профессор кафедры «Компьютерные системы» Одесского национального политехнического университета, Одесса, Украина.

\section{АННОТАЦИЯ}

Актуальность. При построении специализированных и программируемых мобильных систем возникает задача перестройки системы, когда нарушаются условия функционирования системы из-за изменения помехо-сигнальной обстановки или условий работы системы. Любая перестройка цифровых частотно-зависимых компонент системы приводит к возникновению в переходного процесса, длительность которого определяется характеристиками компонент. Традиционный подход к анализу переходного процесса осуществляется при нулевых начальных условиях, однако работа интеллектуальных датчиков специализированных компьютерных систем и перестройка параметров, а также других компонент системы может осуществляться при ненулевых начальных условиях. Это приводит к необходимости большого числа вычислений, что иногда не осуществимо в реальном масштабе времени. В этих условия необходимо оценить длительность переходного процесса до процесса перестройки, чтобы обеспечить работу системы в новых условиях.

Цель. Оценить максимальную длительность переходного процесса при перестройке, определить диапазон и ширину возможных перестроек с учетом обеспечения устойчивости системы.

Метод. В работе усовершенствован косвенный метод оценки длительности переходного процесса по полюсам передаточной функции за счет упрощения разложения иррациональной функции.

Результаты. Проведенные анализ и моделирование позволили получить соотношения для оценки максимальной длительности переходного процесса, определить диапазон и ширину возможных перестроек с учетом обеспечения устойчивости системы. Построены зависимости длительности переходного процесса от относительной частоты среза. Упрощено представление соотношения для определения длительности переходного процесса за счет разложения в степенной ряд.

Выводы. Полученные результаты позволяют оценить верхнюю границу длительности переходного процесса, за счет усовершенствования косвенного метода оценки длительности переходного процесса по полюсам передаточной функции при упрощении разложения иррациональной функции, что дает возможность до начала перестройки при заданной новой относительной частоте среза и порядке компоненты дать прогноз об устойчивости компоненты после перестройки. С практической точки зрения это сокращает объем вычислений и за счет прогноза повышает эффективность работы системы и компоненты специализированной компьютерной системы при заданных критериях качества работы. Результаты применимы при проектировании микропроцессорных компонент компьютерных систем.

КЛЮЧЕВЫЕ СЛОВА: фильтрация, интеллектуальные датчики, анализ длительности переходного процесса, перестройка параметров фильтра.

\section{ЛІТЕРАТУРА / ЛИТЕРАТУРА}

1. Борисова Л. Ф. Методы анализа и расчета переходных процессов в электрических цепях / Л. Ф. Борисова // Международный журнал прикладных и фундаментальных исследований. - 2014, - № 6. - С. 113-114.
2. Бородянский И. М. Применение рекурсивных КИХфильтров для подавления помех при автоматическом контроле сопротивления изоляции / И. М. Бородянский, И. И. Турулин // Известия ЮФУ. Технические науки. 2016. - № 10 (183). - С. 99-110.

3. Войтович И. Д. Интеллектуальные сенсоры / И. Д. Войтович, В. М. Корсунский - М. : Интернет-Университет 
Информационных Технологий; БИНОМ. Лаборатория знаний, 2011. - 624 с.

4. Tuzlukov V. Signal Processing in Radar Systems / V. Tuzlukov. - CRC Press, 2013. - 596 p.

5. Kocon S., Piskorowski J. Digital finite impulse response notch filter with non-zero initial conditions, based on an infinite impulse response prototype filter / S. Kocon, J. Piskorowski // ASC Publications Anal. Chem. - 2014. 86. - P. 3508-3516. dx.doi.org/10.1021/ac404150d

6. Ухина А.В. Повышение эффективности программнотехнических комплексов в АСУ ТП АЭС / А. В. Ухина, А. А. Биленко, В. С. Ситников // Ядерная и радиационная безопасность - 2016. - № 3. - С. 70-76.

7. Попов Д. И. Анализ цифровых систем обработки сигналов / Д. И. Попов // Известия высших учебных заведений. Технические науки. - 2016. - №2 (38). - С. 83-92.

8. Ситніков В. С. Вплив особливостей каскадів цифрового фільтру високого порядку на вихідний шум квантування / В. С. Ситніков, П. В. Ступень, Б. О. Півень // Электротехнические и компьютерные системы. - 2013. №9(85). - С. 97-101.

9. Джиган В. Адаптивная фильтрация сигналов: теория и алгоритмы / В. Джиган. - М. : Техносфера, 2013. - 528 с.

10. Dorf R.C., Bishop R.H. Modern Control Systems / R. C. Dorf, R. H. Bishop. - Prentice Hall, NJ 07458, 2001. $832 \mathrm{p}$.

11. Бесекерский В. А. Теория систем автоматического регулирования / В. А. Бесекерский, Е. П. Попов. - М. : Наука, $1975-768$ с

12. Кузнецов Б. И. Микропроцессорное управление многоканальными системами высокой точности / Б. И. Кузнецов, В. Е. Сергеев, В. М. Чернышев. - К. : Техніка, 1990. $-208 \mathrm{c}$.

13. Иванов В. А. Теория дискретных систем автоматического управления / В. А. Иванов, А. С. Ющенко. - М. : Наука, 1983. $-336 \mathrm{c}$.

14. Kuo B. C. Digital Control Systems / B. C. Kuo. - Holt, Rinehart and Winston, Inc., 1980. - 448 p.
15. Iserman R. Digital Control Systems / R. Iserman. Springer-Verlag, Berlin, Heidelberg, N. York, 1981. $541 \mathrm{p}$.

16. Anderson B.D.O., Bitmead R.R., Johnson C.R. Stability of Adaptive Systems: passivity and averaging analysis / B.D.O. Anderson, R R. Bitmead, C. R. Johnson. - The MIT Press Cambridge, Massachusetts, London, England, 1986. $263 \mathrm{p}$.

17. Schulze K-P., Rehberg K-J. Entwurf von adaptiven Systemen Eine Darstellung fur Ingenieure / K-P. Schulze, K-J. Rehberg. - Veb Verlag Technik, Berlin, 1990. - 448 p.

18. Прикладные математические методы анализа в радиотехнике - Под ред. Г. В. Обрезкова. - М. : Высш. Шк., 1985. - $343 \mathrm{c}$.

19. Методы классической и современной теории автоматического управления. Т.1. Математические модели, динамические характеристики и анализ систем автоматического управления / Под ред. К. П. Пупкова, Н. Д. Егупова - М. : Изд-во МГТУ им. Н. Э. Баумана, 2004. $654 \mathrm{c}$.

20. Журавлев А. Ю. Методика повышения общей устойчивости и коррекции прямых показателей качества переходных процессов в цифровых системах управления / А. Ю. Журавлев, А. В. Павлов, В. О. Журба // Компрессионное и энергетическое машиностроение. - 2012. № 1. - C. 36-40.

21. Кисель А. Г. Анализ длительности переходного процесса при ненулевых начальных условиях / А. Г. Кисель, В. С. Ситников, А. В. Ухина // «Проблеми інформатики та комп'ютерної техніки» (ПІКТ-2017). Праці VI Міжнар. наук.-практ. конф. (Чернівці, Україна, жовтень 5-8, 2017). - C. 100-102.

22. Ухина А. В. Применение компонентов специализированных систем при проведении экспериментальных медико-биологических исследований / А. В. Ухина, В. С. Ситников, В. А. Ситникова // «Датчики, прилади та системи-2017». Тези VI Міжнар. наук.-техн. конф. (Черкаси-Миколаїв-Херсон-Лазурне, Україна, вересень 18 22, 2017). - C. 39-41. 